\title{
A CONVEXITY CONDITION IN BANACH SPACES AND THE STRONG LAW OF LARGE NUMBERS ${ }^{1}$
}

\section{ANATOLE BECK}

Introduction. The strong law of large numbers can be shown under certain hypotheses for random variables which take values in Banach spaces. The general statement reads as follows:

THEOREM. Let $\mathfrak{X}$ be a Banach space and let $\left\{X_{i}\right\}$ be a sequence of

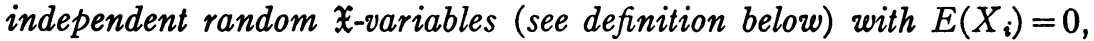
all $i>0$. Under appropriate conditions on $\mathfrak{X}$ and on $\left\{X_{i}\right\}$, we can then assert that $(1 / n) \sum_{i=1}^{n} X_{i}$ converges to 0 in the strong topology of $\mathfrak{X}$ almost surely. ${ }^{2}$

In a recent paper [ 1$]$, this author showed this theorem under the hypotheses that $X$ is uniformly convex and that the variances of $X_{i}$ are uniformly bounded $\left(\operatorname{Var}\left(X_{i}\right)=E\left(\left\|X_{i}\right\|^{2}\right)\right)$. At the same time, an example was given of a space in which the theorem fails. It is now possible, using the methods of [1], to show a necessary and sufficient condition on the Banach space $\mathfrak{X}$ to yield this particular strong law of large numbers.

A Banach space $\mathfrak{X}$ is said to have property (A) if, for every sequence $\left\{X_{i}\right\}$ of independent random $\mathfrak{X}$-variables with $E\left(X_{i}\right)=0$, all $i$, and $\operatorname{Var}\left(X_{i}\right)<M$, all $i$, we have

$$
\frac{1}{n} \sum_{i=1}^{n} X_{i} \rightarrow 0 \quad \text { strongly almost surely. }
$$

A Banach space $\mathfrak{X}$ is said to have property (B) if there exists an integer $k>0$ and an $\epsilon>0$ such that any choice $a_{1}, a_{2}, \cdots, a_{k}$ of elements from $\mathfrak{X}$ with $\left\|a_{i}\right\| \leqq 1$ gives us

$$
\left\| \pm a_{1} \pm a_{2} \pm \cdots \pm a_{k}\right\|<k(1-\epsilon)
$$

for some combination of the + and - signs.

We shall show that these two conditions are equivalent.

1. Definitions. Let $\mathfrak{X}$ be a separable Banach space and let

\footnotetext{
${ }^{1}$ This research was supported by Cornell University under contract with the Office of Naval Research and by the University of Wisconsin under contract number AF 49(638)-868 with the Air Force Office of Scientific Research.

${ }^{2}$ Here and hereafter in this paper unless otherwise specified, all limits are taken as $n \rightarrow \infty$.
}

Presented to the Society, January 23, 1961; received by the editors February 14, 1961. 
$(S, \Sigma, m)$ be a measure space. Then $A$ mapping $X$ from $S$ into $\mathfrak{X}$ is called strongly measurable if $X^{-1}(B)=\{s \mid X(s) \in B\}$ is measurable for every Borel set $B \subset X$. If $X$ is strongly measurable and if $\int_{S}\|X(s)\| m d s$ $<\infty$ then it can be shown that there is a $y \in X$ such that $x^{*}(y)$ $=\int_{S} x^{*}(X(s)) m d s$ for every $x^{*} \in \mathfrak{X}^{*}$. $y$ is defined as the integral of $X$.

A probability space (customarily denoted $(\Omega, B, \operatorname{Pr})$ ) is a measure space of total measure $1 .(\operatorname{Pr}(\Omega)=1$.) A strongly measurable function $X$ from $\Omega$ into $\mathfrak{X}$ is called a random $\mathfrak{X}$-variable, and its integral, if it has one, is called its expectation, $E(X)$. If $X_{1}, \cdots, X_{m}$ are random $\mathfrak{X}$ variables and if for every choice $B_{1}, \cdots, B_{m}$ of Borel sets from $\mathfrak{X}$, we have

$$
\operatorname{Pr}\left\{X_{1} \in B_{1}, \cdots, X_{m} \in B_{m}\right\}=\prod_{i=1}^{m} \operatorname{Pr}\left(X_{i} \in B_{i}\right),
$$

then $X_{1}, \cdots, X_{m}$ are an independent collection of random $\mathfrak{X}$-variables. If, in an infinite collection $\left\{X_{\alpha}, \alpha \in A\right\}$ of random $\mathfrak{X}$-variables, every finite sub-collection is independent, then the infinite collection is said to be independent.

For each random $\mathfrak{X}$-variable $X$, we define $\operatorname{Var}(X)=E\left(\|X-E(X)\|^{2}\right)$ $=\int_{\Omega}\|X(\omega)-E(X)\|^{2} \operatorname{Pr} d \omega$. For each sequence $\left\{X_{i}\right\}$ of random $\mathfrak{X}$ variables, we define

$$
c\left\{X_{i}\right\}=\underset{\Omega}{\operatorname{ess} \sup } \limsup _{n}\left\|\frac{1}{n} \sum_{i=1}^{n} X_{i}\right\| .
$$

A random $\mathfrak{X}$-variable $X$ is called symmetric if there is a measurepreserving mapping $\phi$ of $\Omega$ into $\Omega$ such that $X(\phi(\omega))=-X(\omega)$ for (almost) all $\omega \in \Omega$.

\section{Condition (B) implies condition (A).}

THEOREM 1. Let $\mathfrak{X}$ be a Banach space satisfying condition (B) and let

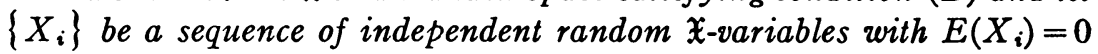
and $\operatorname{Var}\left(X_{i}\right)<M, \quad i=1,2, \ldots$. Then

$$
\frac{1}{n} \sum_{i=1}^{n} X_{i} \rightarrow 0 \quad \text { strongly in } \mathfrak{X} \text { almost surely. }{ }^{3}
$$

Instead of proving Theorem 1, we prove Lemma 2, below, which is the same result under additional hypotheses. The derivation of Theorem 1 from Lemma 2, i.e., the exorcism of the extraneous hypotheses, can be taken verbatim from [1], since the convexity condition is used only in Lemma 2.

I.e., for almost all $\omega \in \Omega$. 
Lemma 2. If $\mathfrak{X}$ satisfies condition (B) and $\left\{X_{i}\right\}$ is a sequence of random $\mathrm{X}$-variables and

(1) the $X_{i}$ are independent,

(2) $E\left(X_{i}\right)=0, i=1,2, \cdots$,

(3) $\left\|X_{i}(\omega)\right\| \leqq 1$, all $i=1,2, \cdots ; \omega \in \Omega$,

(4) $X_{i}$ is symmetric, $i=1,2, \cdots$,

then

$$
\frac{1}{n} \sum_{i=1}^{n} X_{i} \rightarrow 0 \text { sirongly in } \mathfrak{X} \text { almost surely. }
$$

Proof. We will designate the sequences satisfying these hypotheses as being of type 2 .

Then we can read the lemma as saying that if $\mathfrak{X}$ satisfies condition (B) and $\left\{X_{i}\right\}$ is of type 2 , then $c\left\{X_{i}\right\}=0$. If we set $C=C(\mathfrak{X})$ $=\sup \left(c\left\{X_{i}\right\} \mid\left\{X_{i}\right\}\right.$ of type 2$)$, then we are to prove that $C=0$.

We assume, contrarily, that $C \neq 0$. (Note that $c\left\{X_{i}\right\}$ always exists under these hypotheses, and does not exceed 1 , so that $C$ exists and is no greater than 1.) We shall derive a contradiction. Choose any $\eta>0$, and let $\left\{U_{i}\right\}$ be chosen so that $\left\{U_{i}\right\}$ is of type 2, and $c\left\{U_{i}\right\}$ $>C-\eta$. Let $k$ and $\epsilon$ be the numbers given to us in the definition of condition (B), and consider the random $\mathfrak{X}$-variables

$$
V_{i}=\frac{U_{k i}+U_{k i-1}+\cdots+U_{k i-k+1}}{k} .
$$

It is easily seen that $\left\{V_{i}\right\}$ is of type 2, and $c\left\{V_{i}\right\}=c\left\{U_{i}\right\}$. Furthermore, we can show that $E\left(\left\|V_{i}\right\|\right)<1-\epsilon / 2^{k}$. Let $\phi_{i}$ be chosen so that

$$
U_{i}\left(\phi_{i}(\omega)\right)=-U_{i}(\omega), \quad U_{j}\left(\phi_{i}(\omega)\right)=U_{j}(\omega),
$$

all $\omega \in \Omega, i=1,2, \cdots, j \neq i .^{4}$ Then if we look at the $2^{k}$ mappings

$$
\phi_{k i}^{\alpha_{k}} \phi_{k i-1}^{\alpha_{k-1}} \cdots \phi_{k i-k+1}^{\alpha_{1}}
$$

given by the possible choices of $\alpha_{j}=0,1, j=1, \cdots, k$, we see that all these are measure-preserving transformations on $\Omega$, and that for every $\omega \in \Omega$, some one of these, $\Phi_{\omega}$, has the property that

$$
\left\|\sum_{j=k i-k+1}^{k i} U_{j}\left(\Phi_{\omega}(\omega)\right)\right\|=\left\| \pm U_{k i-k+1}(\omega) \pm \cdots \pm U_{k i}(\omega)\right\|<k(1-\epsilon) .
$$

Therefore, if we number these $2^{k}$ mappings as $\Phi_{1}, \Phi_{2}, \cdots, \Phi_{2^{k}}$, we have

4 Possibly this may require a change to an equivalent sequence of random variables in an isomorphic measure space. The truth of this lemma survives such a transplanting. 


$$
\sum_{r=1}^{2 k}\left\|\sum_{j=k i-k+1}^{k i} U_{j}\left(\Phi_{r}(\omega)\right)\right\|<k\left(2^{k}-1\right)+k(1-\epsilon)=k\left(2^{k}-\epsilon\right)
$$

for each $\omega \in \Omega$, and therefore

$$
2^{k} E\left(\left\|\sum_{j=k i-k+1}^{k i} U_{j}\right\|\right)=E\left(\sum_{r=1}^{2 k}\left\|\sum_{j=k i-k+1}^{k i} U_{j}\left(\Phi_{r}(\cdot)\right)\right\|\right)<k\left(2^{k}-\epsilon\right),
$$

so that

$$
E\left(\left\|V_{i}\right\|\right)=\frac{1}{k} E\left(\left\|\sum_{j=k i-k+1}^{k i} U_{j}\right\|\right)<\frac{1}{k} \cdot \frac{k\left(2^{k}-\epsilon\right)}{2^{k}}=1-\frac{\epsilon}{2^{k}} .
$$

Remember that $\epsilon$ and $k$ are constants depending only on the space $\mathfrak{X}$.

Let $t>1 / \eta^{8}$, and for each $i>0$, define

$$
W_{i}=\frac{V_{t i}+V_{t i-1}+\cdots+V_{t i-t+1}}{t} .
$$

We easily see that $\left\{W_{i}\right\}$ is of type 2, and that $c\left\{W_{i}\right\}=c\left\{V_{i}\right\}$. Since $\operatorname{Var}\left(\left\|V_{i}\right\|\right)<1$, all $i=1,2, \cdots$, and the $V_{i}$ are independent, we have

$$
\operatorname{Var}\left(\sum_{j=t i-t+1}^{t i}\left\|\frac{V_{j}}{t}\right\|\right)<\frac{1}{t}
$$

Thus, for each $i>0$,

$$
\begin{aligned}
\operatorname{Pr}\left\{\left\|W_{i}\right\|>1-\frac{\epsilon}{2^{k}}+\eta\right\} & =\operatorname{Pr}\left\{\left\|\sum_{j=t i-t+1}^{t i} \frac{V_{j}}{t}\right\|>1-\frac{\epsilon}{2^{k}}+\eta\right\} \\
& \leqq \operatorname{Pr}\left\{\sum_{j=t i-t+1}^{t i}\left\|\frac{V_{j}}{t}\right\|>1-\frac{\epsilon}{2^{k}}+\eta\right\} \\
& <\frac{1 / t}{\eta^{2}}<\eta,
\end{aligned}
$$

by Chebyshev's inequality. Using the independe: ce of the $W_{i}$, we define

$$
\begin{aligned}
& Y_{i}=W_{i}, \quad Z_{i}=0 \quad \text { if } \quad\left\|W_{i}\right\| \leqq 1-\frac{\epsilon}{2^{k}}+\eta, \\
& Y_{i}=0, \quad Z_{i}=W_{i} \text { if } \quad\left\|W_{i}\right\|>1-\frac{\epsilon}{2^{k}}+\eta,
\end{aligned}
$$


Then $\left\{Y_{i}\right\}$ is of type 2 , and in fact $\left\|Y_{i}(\omega)\right\| \leqq 1-\epsilon / 2^{k}+\eta$, all $\omega \in \Omega, i=1,2, \cdots$, so that $c\left\{Y_{i}\right\} \leqq C\left(1-\epsilon / 2^{k}+\eta\right)$. The sequence $\left\{\left\|Z_{i}\right\|\right\}$ is independent, and since $\left\|Z_{i}(\omega)\right\| \leqq 1$, all $\omega \in \Omega, i=1,2, \ldots$ and $\operatorname{Pr}\left\{Z_{i}=0\right\}>1-\eta$, we have $E\left(\left\|Z_{i}\right\|\right)<\eta$. Thus $c\left\{Z_{i}\right\} \leqq c\left\{\left\|Z_{i}\right\|\right\}$ $\leqq \eta$, by the (real-valued) strong law of large numbers. It is easily seen that $c\left\{W_{i}\right\} \leqq c\left\{Y_{i}\right\}+c\left\{Z_{i}\right\}$, and thus

$$
\begin{aligned}
C-\eta & <c\left\{U_{i}\right\}=c\left\{V_{i}\right\}=c\left\{W_{i}\right\} \\
& \leqq c\left\{Y_{i}\right\}+c\left\{Z_{i}\right\} \\
& \leqq C\left(1-\frac{\epsilon}{2^{k}}+\eta\right)+\eta .
\end{aligned}
$$

Since $C \leqq 1$, we have $C\left(\epsilon / 2^{k}\right)<3 \eta$ for every $\eta>0$ which is impossible if $C>0$. This proves the lemma.

\section{Condition (A) implies condition (B).}

TheOREM 3. Let $\mathfrak{X}$ be a Banach space in which condition (B) fails. Then there is in $\mathfrak{X}$ a sequence $\left\{X_{i}\right\}$ of type 2 such that

$$
c\left\{X_{i}\right\}=1.5
$$

Proof. If $\mathfrak{X}$ fails to meet condition (B), then for every $k$ and $\epsilon$, there are $k$ vectors $a_{1}, \cdots, a_{k}$ in the unit ball of $\mathfrak{X}$ such that $\left\| \pm a_{1} \pm \cdots \pm a_{k}\right\| \geqq k(1-\epsilon)$ for every choice of signs. We now choose any sequences $\left\{\epsilon_{i}\right\}$ and $\left\{\delta_{i}\right\}$ of positive real numbers with $\epsilon_{n} \rightarrow 0$ and $\delta_{n} \rightarrow 0$. We now proceed as follows:

We choose an integer $k_{1}$ with $k_{1}>\left(1-\delta_{1}\right) / \delta_{1}$ and a set of elements $a_{1}^{(1)}, \cdots, a_{k_{1}}^{(1)}$ such that

$$
\left\| \pm a_{1}^{(1)} \pm a_{2}^{(1)} \pm \cdots \pm a_{k_{1}}^{(1)}\right\| \geqq k_{1}\left(1-\epsilon_{1}\right)
$$

for all choices of signs. Then, for each $n>1$, we set

and choose

$$
m_{n}=\sum_{i=1}^{n-1} k_{i}
$$

$$
k_{n}>\frac{1-\delta_{n}}{\delta_{n}} m_{n}
$$

and $k_{n}$ elements $a_{1}^{(n)}, \cdots, a_{k_{n}}^{(n)}$ such that

$$
\left\| \pm a_{1}^{(n)} \pm \cdots \pm a_{k_{n}}^{(n)}\right\| \geqq k_{n}\left(1-\epsilon_{n}\right)
$$

- For definition of type 2, see proof of Lemma 2. 
for all choices of signs. This gives us

$$
\frac{k_{n}}{m_{n+1}}>1-\delta_{n}
$$

and

$$
\frac{m_{n}}{m_{n+1}}<\delta_{n}
$$

For any integer $i$, we have $m_{j}<i \leqq m_{j+1}$ for some value of $j$, i.e., $i=m_{j}+r$, where $1 \leqq r \leqq k_{j}$. Define $b_{i}=a_{r}^{(j)}$. This gives us a sequence $\left\{b_{i}\right\}$ of elements of $\mathfrak{X}$. We define a sequence $\left\{X_{i}\right\}$ of random $\mathfrak{X}$ variables by requiring that the $X_{i}$ be independent and that $\operatorname{Pr}\left\{X_{i}=b_{i}\right\}=\operatorname{Pr}\left\{X_{i}=-b_{i}\right\}=1 / 2$. Then $\left\{X_{i}\right\}$ is a sequence of type 2 , and for each $j$ and each $\omega \in \Omega$, we have

$$
\begin{aligned}
\left\|\frac{1}{m_{j+1}} \sum_{i=1}^{m_{j+1}} X_{i}(\omega)\right\| & \geqq\left\|\frac{1}{m_{j+1}} \sum_{i=m_{j+1}}^{m_{j+1}} X_{i}(\omega)\right\|-\left\|\frac{1}{m_{j+1}} \sum_{i=1}^{m_{j}} X_{i}(\omega)\right\| \\
& \geqq \frac{1}{m_{j+1}}\left\| \pm a_{1}^{(j)} \pm a_{2}^{(j)} \pm \cdots \pm a_{k_{j}}^{(j)}\right\|-\frac{1}{m_{j+1}} m_{j} \\
& \geqq \frac{1}{m_{j+1}} k_{j}\left(1-\epsilon_{j}\right)-\frac{m}{m_{j+1}} \\
& >\left(1-\delta_{j}\right)\left(1-\epsilon_{j}\right)-\delta_{j} .
\end{aligned}
$$

Thus, for every $\omega \in \Omega$,

$$
\limsup _{n}\left\|\frac{1}{n} \sum_{i=1}^{n} X_{i}(\omega)\right\|=1,
$$

so that $c\left\{X_{i}\right\}=1$, as required.

Corollary 4. If $\mathfrak{X}$ is a Banach space, then the constant $C(\mathfrak{X})$, defined in the proof of Lemma 2, can take only the values 0 or 1.

\section{Bibliography}

1. Anatole Beck, Une loi forte des grandes nombres dans des espaces de Banach uniformément convexes, Ann. Inst. H. Poincaré 16 (1958), 35-45.

Cornell University and

UNIVERSITY OF WISCONSIN 